\title{
Nociceptive Neuronal Fc-gamma receptor I is involved in IgG immune complex induced pain in the rat
}

\author{
Haowu Jiang ${ }^{1}$, Xinhua Shen ${ }^{1}$, Zhiyong Chen, Fan Liu, Tao Wang, Yikuan Xie, Chao Ma*
}

\begin{abstract}
Antigen-specific immune diseases such as rheumatoid arthritis are often accompanied by pain and hyperalgesia. Our previous studies have demonstrated that Fc-gamma-receptor type I (Fc $\gamma \mathrm{RI})$ is expressed in a subpopulation of rat dorsal root ganglion (DRG) neurons and can be directly activated by IgG immune complex (IgG-IC). In this study we investigated whether neuronal Fc $\gamma$ RI contributes to antigen-specific pain in the naïve and rheumatoid arthritis model rats. In vitro calcium imaging and whole-cell patch clamp recordings in dissociated DRG neurons revealed that only the small-, but not medium- or large-sized DRG neurons responded to IgG-IC. Accordingly, in vivo electrophysiological recordings showed that intradermal injection of IgG-IC into the peripheral receptive field could sensitize only the $\mathrm{C}$ - (but not A-) type sensory neurons and evoke action potential discharges. Pain-related behavioral tests showed that intradermal injection of IgG-IC dosedependently produced mechanical and thermal hyperalgesia in the hindpaw of rats. These behavioral effects could be alleviated by localized administration of non-specific IgG or an Fc $\gamma \mathrm{RI}$ antibody, but not by mast cell stabilizer or histamine antagonist. In a rat model of antigen-induced arthritis (AIA) produced by methylated bovine serum albumin, Fc $\gamma$ RI were found upregulated exclusively in the small-sized DRG neurons. In vitro calcium imaging revealed that significantly more small-sized DRG neurons responded to IgG-IC in the AIA rats, although there was no significant difference between the AIA and control rats in the magnitude of calcium changes in the DRG neurons. Moreover, in vivo electrophysiological recordings showed that $\mathrm{C}$-nociceptive neurons in the AIA rats
\end{abstract}


exhibited a greater incidence of action potential discharges and stronger responses to mechanical stimuli after IgG-IC was injected to the receptive fields. These results suggest that FcyRI expressed in the peripheral nociceptors might be directly activated by IgG-IC and contribute to antigen-specific pain in pathological conditions.

Key words: Fc-gamma receptor type I, IgG immune complex, Dorsal root ganglion, Pain, Nociceptive neuron

\section{Introduction}

Fc-gamma receptors ( $\mathrm{Fc} \gamma \mathrm{Rs})$, which bind to the $\mathrm{Fc}$ domain of immunoglobulin (Ig) $\mathrm{G}$, are widely expressed in immune cells to regulate adaptive immunity (Nimmerjahn and Ravetch, 2007). Among Fc $\gamma R s$, Fc $\gamma R I$ is the only high-affinity activating receptor for $\operatorname{IgG}$ immune complex (IgG-IC) (Nimmerjahn and Ravetch, 2006, 2008). Recent studies have demonstrated that Fc $\gamma$ RI is also expressed in a subpopulation of dorsal root ganglion (DRG) neurons and co-expressed with nociceptive markers including TRPV1, IB4, CGRP and substance P (Andoh and Kuraishi, 2004; Qu et al., 2011). Moreover, IgG-IC can directly activate neuronal Fc $\gamma$ RI producing an increase in intracellular calcium $\left(\left[\mathrm{Ca}^{2+}\right]_{\mathrm{i}}\right.$ ) (Andoh and Kuraishi, 2004; Qu et al., 2011) and evoking action potential discharges via TRPC3 channel through the Syk-PLC-IP 3 pathway (Qu et al., 2012; Qu et al., 2011). IgG-IC may also induce the release of certain neurotransmitters from DRG neurons, such as substance P (Andoh and Kuraishi, 2004) and CGRP (Harada et al., 2010). These findings suggest a potential role of neuronal Fc $\gamma \mathrm{RI}$ in pain sensation (Wang and Ma, 2016).

Pain and hyperalgesia often accompany immune diseases such as rheumatoid arthritis (RA), which affects millions of people worldwide (Brundtland, 2003; Heiberg and Kvien, 2002; Taylor et al., 2010; Wolfe and Michaud, 2007). During joint inflammation, the peripheral sensory nerve endings innervating the joint were sensitized to mechanical stimuli via the actions of neuropeptides, ion channel ligands, eicosanoids and proteinase-activated receptors, producing pain and hyperalgesia (McDougall et al., 1997). Although it was believed that inflammatory factors play a major role in RA-induced pain, currently available treatments do not always effectively alleviate the pain symptoms in RA patients. Intravenous immunoglobulin (IVIG), typically containing more than $95 \%$ non-specific IgG, may have beneficial effects on specific subgroups of RA patients responded poorly to anti-cytokine blockers or rituximab treatments (Bayry et al., 2007; Katz-Agranov et al., 2015; Pyne et al., 2002). Moreover, the symptoms of experimental antigen-induced arthritis, including pain, 
were ameliorated in a variety of Fc $\gamma \mathrm{R}-k n o c k o u t$ mice, especially Fc $\gamma \mathrm{RI}^{-/-}$mice (Ioan-Facsinay et al., 2002; van Lent et al., 2001). IgG-IC binding to Fc receptors may induce pain through the release of inflammatory cytokines from certain immune cells (Nimmerjahn and Ravetch, 2006, 2008; Pinto et al., 2010; Verri et al., 2008; Verri et al., 2007). However, according to published findings described above, a direct effect of IgG-IC to the FcyRI on nociceptive primary sensory neurons may also involve in the mechanisms of pain in RA and other pathological conditions.

In this study, we tested the direct effects of IgG-IC to primary nociceptive neurons in the naïve rats in vitro and in vivo, and explored the potential role of neuronal FcyRI in the development of pain and hyperalgesia in a rat model of antigen-induced arthritis.

\section{Material and methods}

\subsection{Animals}

Adult female Sprague-Dawley rats (150-180 g, National Institutes for Food and Drug Control, China) were used in this study (Qu et al., 2012; Qu et al., 2011). Rats were housed in temperature $\left(23 \pm 3{ }^{\circ} \mathrm{C}\right)$ and $12 \mathrm{~h} \mathrm{light} / 12 \mathrm{~h}$ dark cycle controlled room with standard rodent chow and water available ad libitum. The experimental protocols were approved by the Institutional Animal Care and Use Committee in Chinese Academy of Medical Sciences, Institute of Basic Medical Sciences. Animals were randomly assigned to treatment or control groups. In order to better exclude the influence of menstrual cycles, at least 8 rats were used for each condition in pain behavioral tests, and were tested at different days in small groups of 2-3 rats. For the other experiments, at least 3 rats were used for each condition and experiments were performed on each rat at different days.

The rat model of Antigen-induced arthritis (AIA) was produced according to the method described previously (Bonnet et al., 2015; Williams et al., 1996; Williams et al., 2001). Briefly, rats were injected subcutaneously (s.c.) with an emulsion $(500 \mu \mathrm{l})$ of equal volumes of methylated bovine serum albumin (mBSA, $0.5 \mathrm{mg}$ ) and Freund's complete adjuvant (0.25 mg Mycobacterium tuberculosis; Sigma-Aldrich, St. Louis, MO, USA) on day 0 and 7 for immunization. Fourteen days after the second immunization, AIA was induced in the rats by an intra-articular injection of mBSA (0.1 $\mathrm{mg}$ in $50 \mu \mathrm{l}$ saline) into the right ankle joint. The left ankle joint was injected with the vehicle (50 $\mu$ l saline).

\subsection{Preparation of IgG immune complex}

IgG-IC was prepared by using ovalbumin (OVA, Sigma-Aldrich) as antigen and rat anti-OVA IgG 
as antibody (Agro-Bio, Atlanta, GA, USA). To avoid the possible toxic and non-specific effects of sodium azide on DRG neurons, the storage buffer of all the IgGs (containing sodium azide) was changed to HEPES buffer (in vitro) or PBS (in vivo) using Zeba ${ }^{\mathrm{TM}}$ spin desalting columns (Thermo Scientific, Rockford, IL, USA) before application. IgG-IC was formed by incubating $10 \mu \mathrm{g} / \mathrm{ml}$ antibody and $6 \mu \mathrm{g} / \mathrm{ml}$ antigen (molar concentration ratio 2:1) for $1 \mathrm{~h}$ at $37{ }^{\circ} \mathrm{C}$, and diluting to the concentrations of $0.001,0.01,0.1,0.3,1$ and $10 \mu \mathrm{g} / \mathrm{ml}$ (measured by the concentrations of $\operatorname{IgG}$ ). In control experiments, the individual components of IC, i.e. OVA at $0.06 \mu \mathrm{g} / \mathrm{ml}$ and rat anti-OVA IgG at $0.1 \mu \mathrm{g} / \mathrm{ml}$, were applied.

\subsection{Pain behavioral test}

Rats were randomly divided into groups. Different concentrations of $\operatorname{IgG}-\mathrm{IC}(0.1,1$ and $10 \mu \mathrm{g} / \mathrm{ml})$, rat anti-OVA IgG $(10 \mu \mathrm{g} / \mathrm{ml})$, OVA $(6 \mu \mathrm{g} / \mathrm{ml})$ or vehicle (PBS), each in a volume of $20 \mu$ were injected intradermally (i.d.) to the plantar skin of hindpaw. The cromoglycate group received a mast cell membrane stabilizer sodium cromoglycate ( $200 \mu \mathrm{g} / \mathrm{paw}$, i.d.; Sigma-Aldrich) 15 min before the IgG-IC injection (Oliveira et al., 2011; Yasuda et al., 2013). The terfenadine group received a nonsedative histamine $\mathrm{H} 1$ receptor antagonist terfenadine (5 $\mathrm{mg} / \mathrm{kg}$, i.p.; Sigma-Aldrich) for 2 consecutive days before the IgG-IC injection (Song et al., 1996). The high-dose IgG group received a mixture of $100 \mu \mathrm{g} / \mathrm{ml}$ non-specific rat IgG and $10 \mu \mathrm{g} / \mathrm{ml} \mathrm{IgG-IC} \mathrm{in} \mathrm{a} \mathrm{volume} \mathrm{of} 20 \mu \mathrm{l}$. The Fc $\gamma \mathrm{RI}$ antibody group received a mixture of $50 \mu \mathrm{g} / \mathrm{ml}$ the affinity-purified rabbit anti-Fc $\gamma \mathrm{RI}$ monoclonal antibody (Sino Biological Inc., China) and $10 \mu \mathrm{g} / \mathrm{ml}$ IgG-IC in a volume of $20 \mu \mathrm{l}$.

Rats were acclimatized to the behavioral testing paradigms for 3 consecutive days prior to baseline testing and were tested for mechanical and thermal hyperalgesia in a blind manner. Pain-related behavioral tests were performed at different time points (1h, 4h, 7h, 10h, 24h) after IgG-IC injection. Post-injection pain responses were measured by assessing withdrawal threshold to mechanical stimuli and latency to radiant heat.

Thermal hyperalgesia was determined according to the method described by Hargreaves et al (Hargreaves et al., 1988) using a radiant thermal stimulator (BME-410C Plantar Test Apparatus, China). In brief, rats were placed individually in transparent Plexiglas chamber $(10 \times 20$ x $20 \mathrm{~cm})$ on an elevated $2 \mathrm{~mm}$-thick-glass platform and allowed to acclimatize for $30 \mathrm{~min}$ before testing. A radiant heat source positioned below the glass was focused onto the plantar surface of the hind paw. The nociceptive end points in the radiant heat test were the characteristic lifting or licking of the hind paw, and the time to the end point was considered the paw withdrawal thermal latency (PWTL). A cut-off time of $20 \mathrm{~s}$ was applied to avoid tissue damage. The test time interval for the same foot was 
$10 \mathrm{~min}$, and the time interval between the tests of two feet was $5 \mathrm{~min}$. The bilateral feet were tested for three times to take the mean latency.

Paw withdrawal mechanical threshold (PWMT) was measured using an electronic von-Frey Anesthesiometer (IITC Life Science, Woodland Hills, CA, USA), which consisted of a hand-held force transducer with a fixed $200 \mu \mathrm{m}$ diameter tungsten wire tip. The electronic von-Frey device was used so that the actual mechanical pain threshold for a single point (site of injection) on the hindpaw plantar surface could be obtained in a relatively short time. For testing, each animal was placed in a Plexiglas chamber $(10 \times 20 \times 20 \mathrm{~cm})$ with a wire grid floor, and allowed to acclimatize for $30 \mathrm{~min}$ before the beginning of the tests in a quiet room. Before paw stimulation, the animals were quiet, without exploratory defecation or urination movements. The investigator was trained to apply the tip perpendicularly to the site of injection on the plantar surface of the hindpaw with a gradual increasing force until a brisk paw withdrawal was elicited. The maximum force $(\mathrm{g})$ applied was automatically recorded when the paw was withdrawn. Lifting of the paw due to normal locomotory behavior was ignored. The average of 3 successive force (g) readings was used as the PWMT (Liu et al., 2012; Vivancos et al., 2004).

For AIA models, tests for PWTL and PWMT were performed at days 0 (baseline), 1, 2, 4, 7, 10, 14 and 21. In addition, the width of ankle between the medial and lateral malleolus was measured with a digital caliper. The force for lifting the hindpaw was measured using the same electronic vonFrey device but with a flat rubber tip ( $\sim \mathrm{mm}$ diameter). The probe was applied perpendicularly to the heel of rat hindpaw with a gradual increasing force until the paw was lifted or withdrawn. The maximum force applied (g) was automatically recorded, and the average of 3 successive force (g) readings was used as the final measurement.

\subsection{Cell dissociation and culture}

DRG neurons were cultured from naïve rats and AIA rats 4 days after challenging. Briefly, ipsilateral L3 to L5 lumbar DRGs were removed and placed in oxygenated complete saline solution (CSS) for cleaning and mincing. The CSS contained (mM): $\mathrm{NaCl} 130, \mathrm{MgSO}_{4} 2, \mathrm{KCl} 3, \mathrm{KH}_{2} \mathrm{PO}_{4}$ 1.25, Dextrose 10, adjusted to $\mathrm{pH} 7.2$ with $\mathrm{NaOH}$. The DRGs were then digested with Collagenase type $2(5 \mathrm{mg} / \mathrm{ml}$; Worthington Biochemical, Lakewood, NJ) in CSS for $30 \mathrm{~min}$. After enzymatic digestion, the ganglia were dissociated into single cells by gentle trituration via a series of heatpolished Pasteur pipettes in cultured medium, then the cells were placed on polyornithine and laminin-coated (Sigma-Aldrich) coverslips. The culture medium contained equal amounts of DMEM and F12 (Gibco, Grand Island, MD, USA) with 10\% FCS (Gibco) and 1\% penicillin and 
streptomycin (Invitrogen). The cells were maintained at $37{ }^{\circ} \mathrm{C}$ in a humidified atmosphere of $95 \%$ air and $5 \% \mathrm{CO}_{2}$ and used within $24 \mathrm{~h}$.

\subsection{Intracellular calcium imaging on dissociated DRG neurons}

Calcium imaging was performed on dissociated DRG neurons as described previously (Qu et al., 2011) from a total of 24 naïve and 4 AIA rats. Briefly, DRG neurons were loaded with $2 \mu \mathrm{M}$ Fura 2acetoxymethyl ester (Invitrogen) in the dark at $37^{\circ} \mathrm{C}$. After loading for $45 \mathrm{~min}$, DRG neurons were washed twice in the HEPES buffer to remove extracellular dye, then placed in a recording chamber continuously perfused with HEPES buffer at a flow rate of $1.5 \mathrm{ml} / \mathrm{min}$ at room temperature. The HEPES buffer contained the following (in $\mathrm{mM}$ ): $\mathrm{NaCl} 145, \mathrm{KCl} 3, \mathrm{CaCl}_{2} 2, \mathrm{MgCl}_{2} 2$, glucose 10 and HEPES 10, adjusted to $\mathrm{pH} 7.4$ with $\mathrm{NaOH}$. Images were recorded using an upright NIKON ECLIPSE Ti microscope equipped with a ratiometric imaging system (Nikon NIS-Elements AR 4.00 .00 , Japan). The ratio of $340 \mathrm{~nm} / 380 \mathrm{~nm}$ fluorescence intensity $\left(\mathrm{R}_{340 / 380}\right)$ within a certain region of interest after background subtraction was used as a relative measure of intracellular calcium concentration $\left(\left[\mathrm{Ca}^{2+}\right]_{\mathrm{i}}\right)$ (Grynkiewicz et al., 1985). Calibration with external standards (Calcium Calibration Buffer Kit, Invitrogen) showed that $\mathrm{R}_{340 / 380}$ increased linearly with $\left[\mathrm{Ca}^{2+}\right]_{\mathrm{i}}$ up to about 1 $\mu \mathrm{M}$ and $\mathrm{R}_{340 / 380}$ of $0.7-1.25$ corresponded to basal $\left[\mathrm{Ca}^{2+}\right]_{\mathrm{i}}$ of $90-180 \mathrm{nM}$. Therefore, the neurons with $\mathrm{R}_{340 / 380}$ at the range of 0.7-1.25 were included in this study.

All agents were dissolved in HEPES buffer and applied locally to the neuronal cell bodies through a micropipette (with a tip diameter of $100 \mu \mathrm{m}$ ) and an 8-channel pressure-controlled drug application system (VC3-8PP, ALA Scientific, Farmingdale, NY, USA). The interval between drug applications was at least $3 \mathrm{~min}$. The proportion of DRG neurons responsive to IC was calculated as the number of IC-responsive neurons divided by all the neurons tested. Neurons were considered capsaicinsensitive if a $10 \mathrm{~s}$ application of $1 \mu \mathrm{M}$ capsaicin evoked an increase in $\mathrm{R}_{340 / 380}$ that was equal or greater than $20 \%$ above baseline. The viability of the neurons was confirmed by the increase in $\left[\mathrm{Ca}^{2+}\right]_{\mathrm{i}}$ produced by a $5 \mathrm{~s}$ application of $50 \mathrm{mM} \mathrm{K}^{+}$at the end of each experiment.

\subsection{In vitro whole-cell patch-clamp recordings}

Whole-cell current-clamp recordings were performed on dissociated DRG neurons from 11 naïve rats as described previously (Qu et al., 2011) using an EPC 10 USB amplifier with Patchmaster software (Heka, Germany). The patch pipettes were pulled from borosilicate glass capillaries (Sutter Instrument; $1.2 \mathrm{~mm}$ outer diameter, $0.69 \mathrm{~mm}$ inner diameter; Novato, CA, USA) with a P97 horizontal puller (Sutter Instrument, Novato, CA, USA). The resistance of the patch pipettes was 3-4 $\mathrm{M} \Omega$ after filled with an internal solution, which contained (in mM): Aspartic Acid 120, KOH 120, 
$\mathrm{KCl} 18, \mathrm{Na}_{2}$-ATP 5, Tris-GTP 1, HEPES 10, EGTA 5, $\mathrm{CaCl}_{2} \bullet 2 \mathrm{H}_{2} \mathrm{O}, \mathrm{MgCl}_{2} \bullet 6 \mathrm{H}_{2} \mathrm{O}$, with pH adjusted to 7.2 using $\mathrm{KOH}$ and had an osmolarity of 290-300 mOsm. The DRG neurons were continuously perfused with the HEPES buffer and all the recordings were performed at room temperature (20$22{ }^{\circ} \mathrm{C}$ ). The series resistance was routinely compensated at 60-80\%. Resting membrane potential (RMP) of each neuron was recorded after stabilization (within 4 min) and only the neuron with a RMP more negative than $-40 \mathrm{mV}$ were included in the study. Capsaicin $(1 \mu \mathrm{M})$ was applied for $10 \mathrm{~s}$ at the end of recordings.

\subsection{In vivo electrophysiological recording from DRG neurons}

A total of 11 naïve rats and 8 AIA rats (4 days after challenging) were used for the in vivo electrophysiological recording. Primary sensory neurons innervating the skin on the hind limb were extracellularly recorded using an in vivo electrophysiology as described previously (Ma et al., 2010; Ma and LaMotte, 2007). A peripheral receptive field (RF) was identified by exploration of the hind limb and application of various handheld stimuli. These included the use of a cotton tip (for innocuous mechanical stimuli), gentle pinching or indentation with a glass probe or von-Frey filaments with fixed tip diameters $(200 \mu \mathrm{m})$ but delivering different bending forces $(10,30,50,70$ and $120 \mathrm{mN}$ ), a temperature-controlled chip-resister heating probe, or ice (for noxious temperature stimuli). Chemicals were injected into the RF, in sequence, each in a volume of $5 \mu 1$, and consisting, in random order, of vehicle $(0.9 \% \mathrm{NaCl})$, and IC $(10 \mu \mathrm{g} / \mathrm{ml})$ using a microsyringe with a $31 \mathrm{G}$ needle (Han et al., 2013; Ma et al., 2012).

Each DRG neuron was classified as $\mathrm{A} \beta-, \mathrm{A} \delta$ - or C-fiber neuron by its cutaneous $\mathrm{RF}$ and conduction velocity. Conduction velocity was obtained by electrically stimulating the cutaneous RF with two wire electrodes or sometimes a saline soaked cotton probe and calculated by dividing the latency of a spike peak by the distance between the stimulation electrode and the soma of the recorded neuron. C-fiber neuron had conduction velocities of $<2 \mathrm{~m} / \mathrm{s}$, whereas $A \delta$ - and $A \beta$-fiber neuron had conduction velocities of 2-12 and >12 m/s, respectively (Ma et al., 2006).

\subsection{Immunohistochemistry}

Immunofluorescent labeling of FcyRI was performed on DRGs from 3 naïve rats and 3 AIA rats (4 days after the challenging) using the method described previously (Qu et al., 2011): Briefly, the L4 and L5 DRGs were harvested from naïve and AIA rats transcardially perfused with PBS followed by $4 \%$ paraformaldehyde, post-fixed in the same fixative for $4 \mathrm{~h}$, and then cryoprotected in $30 \%$ sucrose overnight. The tissue was frozen and sectioned at $12 \mu \mathrm{m}$ thick on a cryostat. Tissue sections were permeabilized with $0.2 \%$ Triton X-100 in PBS for 15 min, then incubated with blocking buffer (3\% 
BSA) for $1 \mathrm{~h}$, followed by overnight incubation at $4{ }^{\circ} \mathrm{C}$ with primary antibodies (rabbit anti-Fc $\gamma \mathrm{RI}$, 1:500, Sino Biological Inc., China; guinea pig anti-PGP9.5, 1:100, LifeSpan BioSciences, Inc., Seattle, WA, USA), and then with the proper secondary antibodies (Alexa Fluor 488-conjugated donkey anti-rabbit, 1:400; Alexa Fluor 594-conjugated donkey anti-guinea pig, 1:500, Jackson ImmunoRresearch Laboratories, West Grove, PA, USA) for $1 \mathrm{~h}$. The slides were then washed in PBS and cover-slipped with VECTASHIELD Mounting Medium with DAPI (Vector Laboratories, Burlingame, CA, USA). The cells were visualized and the images were captured using a laser confocal microscopic imaging system (FV1000 and Olympus FluoView software, Olympus, Japan). The dissociated DRG neurons in adult rats are categorized into small- $(10-30 \mu \mathrm{m})$, medium- $(30-45$ $\mu \mathrm{m})$ and large-sized (>45 $\mu \mathrm{m}$ ) based on somal diameter (White et al., 2005). Taking into account the approximately $10 \%$ decrease in size because of the fixation procedure, DRG neurons were classified as small- (area $<636 \mu \mathrm{m}^{2}$ ), medium- (area 637-1431 $\mu^{2}$ ) and large-sized (area >1431 $\mu \mathrm{m}^{2}$ ) according to their cross-sectional areas (Qu et al., 2011). At least 12 fields of view (200X) from 3 sections on each ganglion were examined. More than 100 neuronal somata with nucleus profiles for each condition were counted and the percentiles of immunopositive neurons were reported.

\subsection{Reverse transcription and quantitative RT-PCR}

Ipsilateral L3 to L5 lumbar DRGs were harvested from 3 naïve rats, 3 sensitized rats (immunized but not challenged), and a total of 15 AIA rats at day $1,2,4,7$, and 14 after challenging ( $\mathrm{n}=3$ rats for each time point) and flash-frozen in liquid nitrogen. Total RNAs were extracted using Trizol reagent (Invitrogen) and reverse transcribed using PrimeScript ${ }^{\mathrm{TM}}$ RT Master Mix (Takara, Japan) according to the manufacturer's instructions. Quantitative RT-PCR (qRT-PCR) was performed on a Bio-Rad CFX96 machine using SYBR Premix Ex Taq (Takara, Japan). The primers used were as follows: CD64 forward, 5'-AGT TGG AGC TAT TTG GTC CCC AGTC-3' and reverse, 5'-GCT AAG GTC CAG GGT CAC CTGA-3'; $\beta$-Tubulin forward, 5'-GTC CGC CTG CCT CTT CGT CTC-3' and reverse, 5'-TTG CCA GCA CCA CTC TGA CCG AA-3'. The expression levels of the target genes were quantified relative to the level of $\beta$-Tubulin gene expression using the $2^{-\Delta \Delta C T}$ method. Real-time PCR experiments for each gene were performed on three separate occasions.

\subsection{Western blotting}

Ipsilateral L3 to L5 lumbar DRGs were harvested from 3 naïve rats, 3 sensitized rats, and 15 AIA rats at day $1,2,4,7$, and 14 after challenging ( $\mathrm{n}=3$ rats for each time point) and then homogenized in the RIPA lysis buffer (CWBio, Beijing, China) containing a mixture of protease inhibitors (CWBio). The protein concentrations of the lysate were measured using Pierce ${ }^{\mathrm{TM}}$ BCA Protein Assay 
kit (Thermo Scientific, Rockford, IL, USA). Equal amounts of protein samples were loaded and separated in 10\% SDS-PAGE gel and subsequently transferred to PVDF membranes (GE Healthcare, Pittsburgh, PA, USA). After blocked in PBST with 5\% nonfat dry milk for $2 \mathrm{~h}$ at room temperature, the membranes were incubated overnight at $4{ }^{\circ} \mathrm{C}$ with rabbit anti-Fc $\gamma \mathrm{RI}(1: 1000$, Sino Biological Inc., China), or mouse anti- $\beta$-Tubulin $(1: 3000$, CWBio) primary antibodies. The membranes were then incubated with HRP-conjugated goat anti-rabbit (1:5000, ZSGB-Bio, Beijing, China) or HRPconjugated goat anti-mouse (1:5000, ZSGB-Bio) secondary antibodies for $1 \mathrm{~h}$ at room temperature. Signals were finally detected using eECL Western Blot Kit (CWBio), and the bands were visualized with the Amersham Imager 600 (GE Healthcare, Pittsburgh, PA, USA). All Western blot analyses were performed at least 3 times, and consistent results were obtained.

\subsection{Statistical analysis}

Data values were presented as means with standard errors (mean \pm SEM). Statistical analyses were performed using the SPSS software (version 17.0). A Student's t-test was used to test the statistical significance of a difference between mean responses for two groups. Statistical comparisons of differences among three or more groups were made with a one-way analysis of variance (ANOVAs) followed by Scheffe's post hoc test. Chi-square tests were used to compare the incidence of neuronal responses. The criterion for statistical significance was a value of $p<0.05$.

\section{Results}

\subsection{IgG-IC exclusively activates nociceptive sensory neurons in the DRG}

Previous studies demonstrated that the IgG-IC formed by the normal mouse IgG and the affinitypurified rat-anti-mouse IgG could directly activate DRG neurons through the neuronal FcyRI (Qu et al., 2011). In this study, we first repeated the experiments using a more reliable formula of IgG-IC consisting OVA and rat-anti-OVA IgG. The effects of IgG-IC were tested on nociceptive DRG neurons using calcium imaging, whole-cell patch-clamp recordings in vitro and extracellular electrophysiology in vivo.

First, we validated the dose-dependent effects of $\mathrm{IgG}-\mathrm{IC}$ on the $\left[\mathrm{Ca}^{2+}\right]_{\mathrm{i}}$ in dissociated DRG neurons (Fig. 1A-C). IgG-IC applied at different concentrations $(0.01,0.1,0.3$, and $1 \mu \mathrm{g} / \mathrm{ml}$, except $0.001 \mu \mathrm{g} / \mathrm{ml})$ caused a significant $\left[\mathrm{Ca}^{2+}\right]_{\mathrm{i}}$ increase in small-sized DRG neurons. Among the 5 concentrations tested, $0.1 \mu \mathrm{g} / \mathrm{ml}$ IgG-IC evoked the largest magnitude of change in $\left[\mathrm{Ca}^{2+}\right]_{\mathrm{i}}$ (measured by $\left.\Delta \mathrm{R}_{340} / 380\right)$ (Fig. 1B), and activated the highest percentage (21.2\%) of neurons (Fig. 1C). 
Therefore the concentration of $0.1 \mu \mathrm{g} / \mathrm{ml} \mathrm{IgG-IC} \mathrm{was} \mathrm{used} \mathrm{in} \mathrm{the} \mathrm{following} \mathrm{in} \mathrm{vitro} \mathrm{experiments.}$ Interestingly, only a few medium-sized neurons (3/65) and none of the large-sized neurons $(0 / 34)$ tested responded to IgG-IC (Fig. 1D). Moreover, IgG-IC responsibility was observed in both capsaicin-sensitive $(22.2 \%, 18 / 81)$ and capsaicin-insensitive neurons $(18.5 \%, 10 / 54)$.

In control experiments, bath application of rat anti-OVA IgG $(0.1 \mu \mathrm{g} / \mathrm{ml}$, Fig. $1 \mathrm{G})$ or OVA $(0.06$ $\mu \mathrm{g} / \mathrm{ml}$, Fig. $1 \mathrm{H})$ alone failed to evoke any significant changes in the basal $\left[\mathrm{Ca}^{2+}\right]_{\mathrm{i}}$, whereas a subsequent application of IgG-IC induced a large increase in $\left[\mathrm{Ca}^{2+}\right]_{\mathrm{i}}$ in the same neuron (Fig. $1 \mathrm{G}, \mathrm{H}$ and I). Furthermore, pretreatment with high-concentration of nonspecific rat $\operatorname{IgG}(1 \mu \mathrm{g} / \mathrm{ml}, 45 \mathrm{~min})$ significantly reduced the proportion of IgG-IC-responsive neurons (Fig. 1E) and the magnitude of IgG-IC-induced $\left[\mathrm{Ca}^{2+}\right]_{\mathrm{i}}$ change (Fig. 1F).

Next, whole-cell current clamp recordings were obtained from dissociated DRG neurons. Bath application of $\mathrm{IgG}-\mathrm{IC}(0.1 \mu \mathrm{g} / \mathrm{ml})$ produced a significant depolarization of the resting membrane potential (RMP, average change $15.8 \pm 2.8 \mathrm{mV}$ ) in $23.7 \%(9 / 38)$ of small-sized dissociated DRG neurons tested (Fig. 2B and C). Moreover, among the 9 responsive neurons, 3 exhibited action potential (AP) discharges during the application of IgG-IC (Fig. 2A). In contrast, none of the medium- (0/19) or large-sized (0/12) neurons responded to IgG-IC (Fig. 2B and C).

Last, in vivo extracellular recordings were performed in $12 \mathrm{~A} \beta-, 6 \mathrm{~A} \delta$ - and $23 \mathrm{C}$-type DRG neurons innervating the skin around ankle joint on the hind limb. Again, injection of IgG-IC (10 $\mu \mathrm{g} / \mathrm{ml}$ ) into the RF failed to produce a response in any of the A $\beta$ - and A $\delta$-type neurons, but evoked action potential discharges in $8.7 \%$ (2/23) C-neurons tested (both were C-mechanoheat nociceptive neurons, see Fig. 3A-E for examples). IgG-IC also induced a decrease of mechanical threshold (minimum force of von Frey filament to induce action potentials) in additional $4 \mathrm{C}$-nociceptive neurons (1 C-mechanoheat and $3 \mathrm{C}$-mechanosensitive neurons). Therefore the total percentage of IgG-IC responsive C-neurons was $26.1 \%$ (6/23). In these 6 responsive C-neurons, IgG-IC induced a significant decrease in the mechanical threshold and a significant increase in the numbers of action potentials evoked by $30 \mathrm{mN}$ and $50 \mathrm{mN}$ stimuli (see Fig. 3D for example, Fig. 7E and F for summary, respectively). One $\mathrm{C}$-mechanoheat neuron displayed stronger responses to heat stimuli $\left(38\right.$ to $51{ }^{\circ} \mathrm{C}$, 5 s) after IgG-IC injection (Fig. 3C).

Therefore the IgG-IC induced neuronal responses were exclusively in small-sized nociceptive DRG neurons, both in vitro and in vivo.

3.2. IgG-IC induced mechanical and thermal hyperalgesia in naïve rats

We accessed the behavioral effects of IgG-IC in the naïve rats. Intra-plantar injection of IgG-IC 
induced dose-dependent mechanical and thermal hyperalgesia (Fig. 4C and D), whereas injection of the vehicle (PBS), antigen (OVA) or antibody (rat anti-OVA IgG) alone did not evoke any significant mechanical or thermal hyperalgesia as compared with the baseline (Fig. 4A and B). After injection of $10 \mu \mathrm{g} / \mathrm{ml}$ IgG-IC, the mechanical withdrawal threshold or thermal withdrawal latency were significantly decreased from the first hour, peaked at around $4 \mathrm{~h}$, and subsided within $24 \mathrm{~h}$ (Fig. 4CD). Therefore, the concentration of $10 \mu \mathrm{g} / \mathrm{ml}$ was chosen for the following experiments.

In order to further investigate the potential role of neuronal Fc $\gamma \mathrm{RI}$ in IgG-IC-induced hyperalgesia, the mast cell stabilizer sodium cromoglycate, the H1R blocker terfenadine, a high dose of nonspecific rat IgG $(100 \mu \mathrm{g} / \mathrm{ml}$ non-specific rat IgG mixed with $10 \mu \mathrm{g} / \mathrm{ml}$ IgG-IC), or an affinitypurified rabbit anti-Fc $\gamma R$ I monoclonal antibody $(50 \mu \mathrm{g} / \mathrm{ml}$ antibody mixed with $10 \mu \mathrm{g} / \mathrm{ml}$ IgG-IC) were applied. We observed that the IgG-IC-induced hyperalgesia could be significantly alleviated by the nonspecific IgG or FcyRI monoclonal antibody, but not by sodium cromoglycate or terfenadine (Fig. 4E and F).

\subsection{AIA induced upregulation of Fc $\gamma$ RI in the DRG}

One day after the challenging with intra-articular injection of mBSA in the sensitized rats, the ipsilateral hindpaw swelled remarkably on the ankle joint and lasted for about a week (Fig. 5A). The force for paw-lifting (Fig. 5B), paw withdrawal mechanical threshold (PWMT, Fig. 5C) and paw withdrawal thermal latency (PWTL, Fig. 5D) also significantly decreased, peaking in day 1 to 4, and then gradually returned to control levels in about 2 weeks. We examined the mRNA and protein levels of Fc $\gamma$ RI in the L3-5 DRGs of AIA rats. qRT-PCR revealed that the levels of Fc $\gamma$ RI mRNA were significantly upregulated on day 0 (i.e. sensitized rats), peaking on day 1 , and returned back to base levels by day 7 (Fig. 6A). Western blot showed that the Fc $\gamma$ RI protein level was significant upregulated on day 0, 1, 2, 4 and 7 (peaking on day 1-4) (Fig. 6B). Moreover, immunofluorescent staining showed that Fc $\gamma$ RI was significantly upregulated only in small-sized DRG neurons, but not in large- or medium-sized neurons (Fig. 6C and D).

\subsection{Primary nociceptors in the AIA rats showed enhanced responses to IgG-IC}

To explore the functional significance of upregulated neuronal Fc $\gamma \mathrm{RI}$ in the AIA model, in vitro calcium imaging and in vivo electrophysiology recordings were performed on DRG neurons from AIA rats. Calcium imaging on dissociated DRG neurons revealed significantly more neurons (all small-sized) responsive to IgG-IC in the AIA rats (Fig. 7A), though the mean magnitude of IgG-ICinduced $\left[\mathrm{Ca}^{2+}\right]_{\mathrm{i}}$ change in the AIA group was not significantly different from the naïve group (Fig. 7B). Interestingly, the proportion of capsaicin-sensitive DRG neurons responding to IgG-IC was also significantly increased in the AIA group (39.1\%) compared with the naïve group (22.2\%, Fig. 7C). 
In vivo extracellular recordings demonstrated that IgG-IC injection to the RF evoked action potential discharges in $38.5 \%$ (10/26) C-nociceptive neurons from the AIA rats, which was significantly higher percentage than what was found in the naïve rats (8.7\%, 2/23) (Fig. 7D). Moreover, as observed in the naïve rats, IgG-IC also induced a significant reduction in the mechanical threshold and increased the number of action potentials evoked by mechanical stimuli of $30 \mathrm{mN}$ and $50 \mathrm{mN}$ forces (Fig. 7E and F) in the 9 C-nociceptive neurons tested. When compared together with the naïve groups, all the values from the AIA rats before or after IgG-IC injection were significantly different from the corresponding values from the naïve rats (Fig. 7E and F).

\section{Discussion}

Previous studies demonstrated that IgG-IC could directly activate DRG neurons through the neuronal Fc $\gamma$ RI (Andoh and Kuraishi, 2004; Qu et al., 2011). In this study, we first confirmed these results with a new formula of IgG-IC (made with OVA and rat anti-OVA IgG). Moreover, we found that IgG-IC exclusively activated small-sized nociceptive DRG neurons, but not large- or mediumsized DRG neurons. The results from in vitro calcium imaging and whole-cell patch clamp recordings highly agreed with each other in terms of the percentage of small-sized neurons responding to IgG-IC (21.2\% vs. $23.7 \%$, respectively). In accord to the in vitro data, in vivo extracellular recordings revealed that $26.1 \%$ of C-nociceptive neurons (and none of the A-type neurons) were activated or sensitized by IgG-IC. Although Fc $\gamma$ RI was found expressed in all size categories of primary sensory neurons (Qu et al., 2011), only the small-sized DRG neurons can be activated by IgG-IC. These observations may indicate a role of neuronal Fc $\gamma$ RI in pain sensation while the underlying mechanisms are obscure.

Fc $\gamma \mathrm{RI}$ is not a ligand gated or G-protein-coupled receptor. As the only high-affinity activating receptor for IgG-IC, Fc $\gamma$ RI belongs to the immunoglobulin superfamily, which consists of a ligandbinding $\alpha$-chain and a signal-transducing FcR $\gamma$-chain with an immunoreceptor tyrosine-based activation motif (ITAM) sequence in its cytoplasmic tail (Nimmerjahn and Ravetch, 2007, 2008; van der Poel et al., 2011). In immune cells, after crosslinking of Fc $\gamma$ RI by IgG-IC, the signaling pathways initiation occurs with tyrosine phosphorylation of the ITAMs by a kinase of the SRC family, which leads to the subsequent recruitment of spleen tyrosine kinase (SyK) family kinases, followed by the activation of various downstream targets including the activation of the phospholipase C (PLC) which are critical for increase in intracellular calcium $\left(\left[\mathrm{Ca}^{2+}\right]_{\mathrm{i}}\right)$ (Nimmerjahn and Ravetch, 2007, 2008). Our previous study demonstrated that in rat small-sized DRG neurons, the activation of Fc $\gamma$ RI by IgG-IC stimulates Syk. Activated Syk leads to the activation of PLC, which subsequently 
hydrolyzes the phosphatidylinositol 4,5-bisphosphate to generate diacylglycerol (DAG) and inositol triphosphate (IP3). IP3 induces $\mathrm{Ca}^{2+}$ release from internal stores through binding to IP3 receptors in endoplasmic reticulum and in turn activates the nonselective cation channel TRPC3, resulting in further $\mathrm{Ca}^{2+}$ influx and membrane depolarization (Qu et al., 2012). However, it was not clear why the IgG-IC induced responses were only observed in a subpopulation of small-diameter, C-type nociceptive DRG neurons, as shown in our results in vitro and in vivo. In supplementary data (Fig. S1), we provided additional data showing that TRPC3 was highly expressed in small-sized DRG neurons, but could also be detected in a few medium- and large-sized DRG neurons. Our previous publication demonstrated that TRPC3 likely behaves as a store-operated channel (SOC) in Fc $\gamma$ RItriggered signaling in the small-sized DRG neurons (Qu et al., 2012). In different sized DRG neurons, the channel configurations and calcium-regulating mechanisms including store-operated $\mathrm{Ca}^{2+}$ entry (Lu et al., 2006) were different, which may be lead to the differential responses in small- vs. largersized neurons to IgG-IC. In addition, pervious studies found that TRPC 3 mRNAs was almost exclusively expressed in nonpeptidergic isolectin B4 (IB4)-positive small-sized neurons (Elg et al., 2007) or C-fiber nociceptors (Alkhani et al., 2014) using in situ hybridization. Taken together, it might be possible that the neuronal specificity of IgG-IC induced responses is due to the coexpression of Fc $\gamma$ RI, TRPC3, and related calcium-regulating pathways (Lu et al., 2006) in a distinct subpopulation of small-sized DRG neurons. The mechanisms of neuronal specificity of IgG-IC responsibility will need further investigation in the future.

In the present study, we observed that the intra-plantar injection of IgG-IC, but not the antigen or antibody alone, dose-dependently induced mechanical and thermal hyperalgesia. In addition, the IgG-IC-induced mechanical and thermal hyperalgesia could be significantly alleviated by a high dose of nonspecific rat IgG or an Fc $\gamma \mathrm{RI}$ antibody, but not by the mast cell stabilizer sodium cromoglycate or the H1R blocker terfenadine. In accord to these behavioral results, we also found that pretreatment with high-concentration of non-specific $\operatorname{IgG}$ attenuated the IC-induced $\left[\mathrm{Ca}^{2+}\right]_{\mathrm{i}}$ increase. These results provided further support for our hypothesis that neuronal Fc $\gamma \mathrm{RI}$ is involved in IgG-IC induced pain. The blocking effect of nonspecific (monomeric) IgG is likely due to its high affinity to FcyRI, resulting a competition between monomeric IgG and IgG-IC (Nimmerjahn and Ravetch, 2006, 2008), which greatly reduces the probability of IgG-IC binding to Fc $\gamma \mathrm{RI}$ expressed in nerve terminals in the peripheral tissue. Monomeric IgG and specific antibodies may serve as potential blockers to neuronal Fc $\gamma \mathrm{RI}$ for the treatment of IgG-IC induced pain.

Fc $\gamma \mathrm{RI}$ is widely expressed in immune cells including monocytes, macrophages, and dendritic cells (DCs) (van der Poel et al., 2011). Although a mast cell stabilizer and a H1R blocker were tested in this study, we cannot exclude the involvements of other immune cells in the hyperalgesia induced by 
IgG-IC. Previous studies indicated that IgG-IC binding to $\mathrm{Fc}$ receptors including Fc $\gamma \mathrm{RI}$ in the peripheral tissue might indirectly activate small-sized DRG neurons (small-caliber fibers) through the release of pro-inflammatory cytokines from immune cells in cases of AIA (Nimmerjahn and Ravetch, 2006, 2008; Pinto et al., 2010; Verri et al., 2008; Verri et al., 2007). In this study we demonstrated that neuronal Fc $\gamma$ RI could also be directly activated by IgG-IC, so there should be at least two different ways to activate small-sized nociceptive DRG neurons by IgG-IC. Future studies using neuronal-specific Fc $\gamma$ RI knockout animals may be required to determine the role of neuronal Fc $\gamma R I$ in IgG-IC induced pain.

During inflammation, cytokines may lead to Fc $\gamma R$ I reorganization on the membrane and/or conformational changes, resulting in preferred binding to IgG-IC (Bracke et al., 2000; Koenderman et al., 1993; van der Poel et al., 2010). In addition, de novo synthesis of free FcyRI caused by cytokine leads to upregulated receptor levels within several hours (Pearse et al., 1991; Pricop et al., 2001; Tridandapani et al., 2003). RA is a typical autoimmune disease caused by self-antibodies against the joint tissue antigen forming immune complexes. In most RA patients, large amounts of IgG-ICs present in the joint and appear to be the major pathogenic factor that responsible for the initiation and persistence of the inflammatory reaction resulting the destruction of the cartilage (Cooke, 1985). Nociceptive nerve terminals are distributed throughout the joint, including the capsule, ligaments, menisci, periosteum and subchondral bone (Freeman and Wyke, 1967; Mach et al., 2002; Marinozzi et al., 1991; McDougall et al., 1997; O'Connor, 1984; Serre et al., 1999). In this study, we found that both the mRNA and protein levels of Fc $\gamma$ RI were significantly upregulated in the DRG of AIA rats. The timing of neuronal Fc $\gamma$ RI upregulation (day 1-4 after challenging) also agreed with the peak time of pain and hyperalgesia in the AIA animals. Moreover, immunohistochemistry staining revealed that Fc $\gamma$ RI was significantly upregulated only in smallsized DRG neurons. Finally, both in vitro calcium imaging and in vivo electrophysiological recordings revealed an enhanced response to IgG-IC exclusively in small-sized, C-nociceptive DRG neurons from the AIA rats. IgG-IC not only evoked action potential discharges in more nociceptive neurons, but also induced more robust neuronal sensitizations in the AIA rats as compared to the naïve rats (Fig. 7D-F). Taken together, our results suggested that nociceptive neuronal Fc $\gamma$ RI could be directly activated by IgG-IC during joint inflammation and might involve in the development of pain and hyperalgesia in RA.

\section{Conflict of interest statement}

The authors have no conflicts of interest to declare. 


\section{Acknowledgements}

This work was supported by grants from National Science Foundation of China \#81271239 and \#91632113 (C.M.), the IBMS/CAMS Dean's Fund \#2011RC01 (C.M.), the PUMC Youth Fund (X.S., and C.M.), and the CAMS Innovation Fund for Medical Sciences (CIFMS).

\section{References}

Alkhani, H., Ase, A.R., Grant, R., O'Donnell, D., Groschner, K., Seguela, P., 2014. Contribution of TRPC3 to store-operated calcium entry and inflammatory transductions in primary nociceptors. Mol Pain 10, 43.

Andoh, T., Kuraishi, Y., 2004. Direct action of immunoglobulin G on primary sensory neurons through Fc gamma receptor I. FASEB journal : official publication of the Federation of American Societies for Experimental Biology 18, $182-184$.

Bayry, J., Lacroix-Desmazes, S., Kazatchkine, M.D., Kaveri, S.V., 2007. Monoclonal antibody and intravenous immunoglobulin therapy for rheumatic diseases: rationale and mechanisms of action. Nature clinical practice. Rheumatology 3, 262-272.

Bonnet, C.S., Williams, A.S., Gilbert, S.J., Harvey, A.K., Evans, B.A., Mason, D.J., 2015. AMPA/kainate glutamate receptors contribute to inflammation, degeneration and pain related behaviour in inflammatory stages of arthritis. Annals of the rheumatic diseases 74, 242-251.

Bracke, M., Nijhuis, E., Lammers, J.W., Coffer, P.J., Koenderman, L., 2000. A critical role for PI 3-kinase in cytokineinduced Fcalpha-receptor activation. Blood 95, 2037-2043.

Brundtland, G.H., 2003. A WHO Scientific Group on the Burden of Musculoskeletal Conditions at the Start of the New Millennium met in Geneva from 13 to 15 January 2000. Who Tech Rep Ser 919, 1-218.

Cooke, T.D.V., 1985. Mechanisms of Cartilage Degradation - Relation to Choice of Therapeutic Agent. Semin Arthritis Rheu 15, 16-23.

Elg, S., Marmigere, F., Mattsson, J.P., Ernfors, P., 2007. Cellular subtype distribution and developmental regulation of TRPC channel members in the mouse dorsal root ganglion. The Journal of comparative neurology 503, 35-46.

Freeman, M.A., Wyke, B., 1967. The innervation of the knee joint. An anatomical and histological study in the cat. Journal of anatomy 101, 505-532.

Grynkiewicz, G., Poenie, M., Tsien, R.Y., 1985. A new generation of Ca2+ indicators with greatly improved fluorescence properties. J Biol Chem 260, 3440-3450.

Han, L., Ma, C., Liu, Q., Weng, H.J., Cui, Y., Tang, Z., Kim, Y., Nie, H., Qu, L., Patel, K.N., Li, Z., McNeil, B., He, S., Guan, Y., Xiao, B., Lamotte, R.H., Dong, X., 2013. A subpopulation of nociceptors specifically linked to itch. Nat Neurosci 16, 174-182.

Harada, N., Zhao, J., Kurihara, H., Nakagata, N., Okajima, K., 2010. Stimulation of Fc gammaRI on primary sensory neurons increases insulin-like growth factor-I production, thereby reducing reperfusion-induced renal injury in mice. Journal of immunology 185, 1303-1310.

Hargreaves, K., Dubner, R., Brown, F., Flores, C., Joris, J., 1988. A new and sensitive method for measuring thermal nociception in cutaneous hyperalgesia. Pain 32, 77-88.

Heiberg, T., Kvien, T.K., 2002. Preferences for improved health examined in 1,024 patients with rheumatoid arthritis: pain has highest priority. Arthritis and rheumatism 47, 391-397.

loan-Facsinay, A., de Kimpe, S.J., Hellwig, S.M.M., van Lent, P.L., Hofhuis, F.M.A., van Ojik, H.H., Sedlik, C., da Silveira, S.A., Gerber, J., de Jong, Y.F., Roozendaal, R., Aarden, L.A., van den Berg, W.B., Saito, T., Mosser, D., Amigorena, S., Izui, S., van Ommen, G.J.B., van Vugt, M., van de Winkel, J.G.J., Verbeek, J.S., 2002. Fc-gamma RI (CD64) contributes substantially to severity of arthritis, hypersensitivity responses, and protection from bacterial infection. Immunity 16 , 391-402.

Katz-Agranov, N., Khattri, S., Zandman-Goddard, G., 2015. The role of intravenous immunoglobulins in the treatment of rheumatoid arthritis. Autoimmunity reviews 14, 651-658.

Koenderman, L., Hermans, S.W., Capel, P.J., van de Winkel, J.G., 1993. Granulocyte-macrophage colony-stimulating factor induces sequential activation and deactivation of binding via a low-affinity IgG Fc receptor, hFc gamma RII, on 
human eosinophils. Blood 81, 2413-2419.

Liu, M., Zhou, L.L., Chen, Z.W., Hu, C.B.A., 2012. Analgesic effect of iridoid glycosides from Paederia scandens (LOUR.) MERRILL (Rubiaceae) on spared nerve injury rat model of neuropathic pain. Pharmacol Biochem Be 102, 465-470.

Lu, S.G., Zhang, X.L., Gold, M.S., 2006. Intracellular calcium regulation among subpopulations of rat dorsal root ganglion neurons. J Physiol-London 577, 169-190.

Ma, C., Donnelly, D.F., LaMotte, R.H., 2010. In vivo visualization and functional characterization of primary somatic neurons. J Neurosci Meth 191, 60-65.

Ma, C., Greenquist, K.W., LaMotte, R.H., 2006. Inflammatory mediators enhance the excitability of chronically compressed dorsal root ganglion neurons. J Neurophysiol 95, 2098-2107.

Ma, C., LaMotte, R.H., 2007. Multiple sites for generation of ectopic spontaneous activity in neurons of the chronically compressed dorsal root ganglion. J Neurosci 27, 14059-14068.

Ma, C., Nie, H., Gu, Q., Sikand, P., Lamotte, R.H., 2012. In vivo responses of cutaneous C-mechanosensitive neurons in mouse to punctate chemical stimuli that elicit itch and nociceptive sensations in humans. J Neurophysiol 107, 357363.

Mach, D.B., Rogers, S.D., Sabino, M.C., Luger, N.M., Schwei, M.J., Pomonis, J.D., Keyser, C.P., Clohisy, D.R., Adams, D.J., O'Leary, P., Mantyh, P.W., 2002. Origins of skeletal pain: sensory and sympathetic innervation of the mouse femur. Neuroscience 113, 155-166.

Marinozzi, G., Ferrante, F., Gaudio, E., Ricci, A., Amenta, F., 1991. Intrinsic innervation of the rat knee joint articular capsule and ligaments. Acta anatomica 141, 8-14.

McDougall, J.J., Bray, R.C., Sharkey, K.A., 1997. Morphological and immunohistochemical examination of nerves in normal and injured collateral ligaments of rat, rabbit, and human knee joints. The Anatomical record 248, 29-39.

Nimmerjahn, F., Ravetch, J.V., 2006. Fcgamma receptors: old friends and new family members. Immunity 24, $19-28$.

Nimmerjahn, F., Ravetch, J.V., 2007. Fc-receptors as regulators of immunity. Adv Immunol 96, 179-204.

Nimmerjahn, F., Ravetch, J.V., 2008. Fc gamma receptors as regulators of immune responses. Nat Rev Immunol 8, 34-47.

O'Connor, B.L., 1984. The mechanoreceptor innervation of the posterior attachments of the lateral meniscus of the dog knee joint. Journal of anatomy 138 ( Pt 1), 15-26.

Oliveira, S.M., Drewes, C.C., Silva, C.R., Trevisan, G., Boschen, S.L., Moreira, C.G., de Almeida Cabrini, D., Da Cunha, C., Ferreira, J., 2011. Involvement of mast cells in a mouse model of postoperative pain. Eur J Pharmacol 672, 88-95.

Pearse, R.N., Feinman, R., Ravetch, J.V., 1991. Characterization of the promoter of the human gene encoding the highaffinity IgG receptor: transcriptional induction by gamma-interferon is mediated through common DNA response elements. Proc Natl Acad Sci U S A 88, 11305-11309.

Pinto, L.G., Cunha, T.M., Vieira, S.M., Lemos, H.P., Verri, W.A., Jr., Cunha, F.Q., Ferreira, S.H., 2010. IL-17 mediates articular hypernociception in antigen-induced arthritis in mice. Pain 148, 247-256.

Pricop, L., Redecha, P., Teillaud, J.L., Frey, J., Fridman, W.H., Sautes-Fridman, C., Salmon, J.E., 2001. Differential modulation of stimulatory and inhibitory Fc gamma receptors on human monocytes by Th1 and Th2 cytokines. Journal of immunology 166, 531-537.

Pyne, D., Ehrenstein, M., Morris, V., 2002. The therapeutic uses of intravenous immunoglobulins in autoimmune rheumatic diseases. Rheumatology (Oxford) 41, 367-374.

Qu, L.T., Li, Y.M., Pan, X.H., Zhang, P., LaMotte, R.H., Ma, C., 2012. Transient Receptor Potential Canonical 3 (TRPC3) Is Required for IgG Immune Complex-Induced Excitation of the Rat Dorsal Root Ganglion Neurons. Journal of Neuroscience 32, 9554-9562.

Qu, L.T., Zhang, P., LaMotte, R.H., Ma, C., 2011. Neuronal Fc-gamma receptor I mediated excitatory effects of IgG immune complex on rat dorsal root ganglion neurons. Brain Behav Immun 25, 1399-1407.

Serre, C.M., Farlay, D., Delmas, P.D., Chenu, C., 1999. Evidence for a dense and intimate innervation of the bone tissue, including glutamate-containing fibers. Bone 25, 623-629.

Song, C., Earley, B., Leonard, B.E., 1996. Behavioural and immunological effects of the antihistamine terfenadine in olfactory bulbectomized rats. Eur Neuropsychopharmacol 6, 157-162.

Taylor, P., Manger, B., Alvaro-Gracia, J., Johnstone, R., Gomez-Reino, J., Eberhardt, E., Wolfe, F., Schwartzman, S., Furfaro, N., Kavanaugh, A., 2010. Patient perceptions concerning pain management in the treatment of rheumatoid arthritis. The Journal of international medical research 38, 1213-1224.

Tridandapani, S., Wardrop, R., Baran, C.P., Wang, Y., Opalek, J.M., Caligiuri, M.A., Marsh, C.B., 2003. TGF-beta 1 suppresses [correction of supresses] myeloid Fc gamma receptor function by regulating the expression and function of the common gamma-subunit. Journal of immunology 170, 4572-4577.

van der Poel, C.E., Karssemeijer, R.A., Boross, P., van der Linden, J.A., Blokland, M., van de Winkel, J.G., Leusen, J.H., 2010. Cytokine-induced immune complex binding to the high-affinity IgG receptor, FcgammaRI, in the presence of monomeric IgG. Blood 116, 5327-5333.

van der Poel, C.E., Spaapen, R.M., van de Winkel, J.G., Leusen, J.H., 2011. Functional characteristics of the high affinity 
IgG receptor, FcgammaRI. Journal of immunology 186, 2699-2704.

van Lent, P.L., Nabbe, K., Blom, A.B., Holthuysen, A.E., Sloetjes, A., van de Putte, L.B., Verbeek, S., van den Berg, W.B., 2001. Role of activatory Fc gamma RI and Fc gamma RIII and inhibitory Fc gamma RII in inflammation and cartilage destruction during experimental antigen-induced arthritis. The American journal of pathology 159, 2309-2320.

Verri, W.A., Guerrero, A.T.G., Fukada, S.Y., Valerio, D.A., Cunha, T.M., Xu, D., Ferreira, S.H., Liew, F.Y., Cunha, F.Q., 2008. IL33 mediates antigen-induced cutaneous and articular hypernociception in mice. P Natl Acad Sci USA 105, $2723-2728$. Verri, W.A., Jr., Cunha, T.M., Parada, C.A., Poole, S., Liew, F.Y., Ferreira, S.H., Cunha, F.Q., 2007. Antigen-induced inflammatory mechanical hypernociception in mice is mediated by IL-18. Brain Behav Immun 21, 535-543.

Vivancos, G.G., Verri, W.A., Cunha, T.M., Schivo, I.R.S., Parada, C.A., Cunha, F.Q., Ferreira, S.H., 2004. An electronic pressure-meter nociception paw test for rats. Brazilian Journal of Medical and Biological Research 37, 391-399.

Wang, T., Ma, C., 2016. Peripheral Nociceptors as Immune Sensors in the Development of Pain and Itch. Advances in experimental medicine and biology 904, 77-85.

White, F.A., Sun, J.H., Waters, S.M., Ma, C., Ren, D.J., Ripsch, M., Steflik, J., Cortright, D.N., LaMotte, R.H., Miller, R.J., 2005. Excitatory monocyte chemoattractant protein-1 signaling is up-regulated in sensory neurons after chronic compression of the dorsal root ganglion. P Natl Acad Sci USA 102, 14092-14097.

Williams, A.S., Camilleri, J.P., Goodfellow, R.M., Williams, B.D., 1996. A single intra-articular injection of liposomally conjugated methotrexate suppresses joint inflammation in rat antigen-induced arthritis. Brit J Rheumatol 35, 719724.

Williams, A.S., Topley, N., Dojcinov, S., Richards, P.J., Williams, B.D., 2001. Amelioration of rat antigen-induced arthritis by liposomally conjugated methotrexate is accompanied by down-regulation of cytokine mRNA expression. Rheumatology (Oxford) 40, 375-383.

Wolfe, F., Michaud, K., 2007. Assessment of pain in rheumatoid arthritis: minimal clinically significant difference, predictors, and the effect of anti-tumor necrosis factor therapy. The Journal of rheumatology 34, 1674-1683.

Yasuda, M., Kido, K., Ohtani, N., Masaki, E., 2013. Mast cell stabilization promotes antinociceptive effects in a mouse model of postoperative pain. J Pain Res 6, 161-166.

\section{Figure Legends}

Fig. 1. IgG-IC induced $\mathrm{Ca}^{2+}$ responses in dissociated DRG neurons. A. Typical calcium responses of small-diameter dissociated DRG neurons to different concentrations of IgG-IC $(0.001 \sim 1 \mu \mathrm{g} / \mathrm{ml})$. B. The magnitude of calcium responses among the responsive neurons. C. The percentage of smallsized neurons responding to different doses of IgG-IC. D. Percentage of small-, medium- and largesized neuronal somata responding to $0.1 \mu \mathrm{g} / \mathrm{ml}$ IgG-IC. E-F. Treatment with non-specific IgG only (nsIgG, $1 \mu \mathrm{g} / \mathrm{ml})$ did not evoke any observable response. Pretreatment with nonspecific rat IgG (1 $\mu \mathrm{g} / \mathrm{ml})$ before the application of IgG-IC $(0.1 \mu \mathrm{g} / \mathrm{ml})$ significantly reduced the percentage $(\mathrm{E})$ of $\mathrm{IgG}-$ IC responsive neurons and the mean magnitude (F) of IgG-IC evoked calcium responses. G-I. Lack of responses when the antibody (rat anti-OVA $\mathrm{IgG}, 0.1 \mu \mathrm{g} / \mathrm{ml}, \mathrm{G}$ ) or antigen (OVA, $0.06 \mu \mathrm{g} / \mathrm{ml}, \mathrm{H}$ ) was applied separately to the DRG neurons (percentile of responsive neurons summarized in I). The numbers of responsive/total neurons tested was given in the parentheses. $* p<0.05$, $* * p<0.01$ vs. $0.1 \mu \mathrm{g} / \mathrm{ml}$ IgG-IC, Chi-square test (C, E and I), one-way ANOVA (B); Student's t-test (F).

Fig. 2. IgG-IC increased membrane excitability of small-sized DRG neurons in vitro. A. Typical current-clamp recordings of IgG-IC $(0.1 \mu \mathrm{g} / \mathrm{ml})$ induced membrane depolarization (left) and AP 
discharges (right) in small-diameter DRG neurons. B. The changes in different sized DRG neurons' RMP before (Vehicle) and during IgG-IC application (IC), and after washout. C. The percentage of different sized DRG neurons responding to $0.1 \mu \mathrm{g} / \mathrm{ml}$ IgG-IC. The numbers of responsive/total neurons tested were given in the parentheses. ${ }^{*} p<0.01$ vs. control, paired t-test (B).

Fig. 3. IgG-IC activated nociceptive DRG neurons in vivo. A. Typical image of a small-sized neuronal soma under recording on the surface of DRG. The dotted lines represented the extracellular recording electrode. B. Conduction velocity $(0.53 \mathrm{~m} / \mathrm{s})$ obtained with an electrical stimulation (arrow) to the peripheral receptive field (red dot in the inset). C. Responses of the C-neuron to heat stimuli (38 to $51{ }^{\circ} \mathrm{C}, 5 \mathrm{~s}$ ) before and after injection of IgG-IC $(10 \mu \mathrm{g} / \mathrm{ml})$ to the receptive field on the hindpaw. D. Responses of the same neuron to mechanical stimuli of different forces $(10,30,50,70$ and $120 \mathrm{mN}$ ) via a von-Frey filament with a $200 \mu \mathrm{m}$ diameter tip for $1 \mathrm{~s}$ before and after IgG-IC injection. E. Action potential discharges evoked in another C-neuron after injection of IgG-IC (10 $\mu \mathrm{g} / \mathrm{ml}$ ), but not vehicle ( $\mathrm{VEH}, 0.9 \% \mathrm{NaCl}$ ) to the receptive field.

Fig. 4. IgG-IC induced mechanical and thermal hyperalgesia in naïve rats. Changes in the paw withdrawal mechanical threshold (PWMT, A, C and E) and the paw withdrawal thermal latency (PWTL, B, D and F) in the ipsilateral hindpaw after intra-plantar injection of IgG-IC or other reagents were summarized. A-B. Injections of the vehicle (PBS), antigen (OVA, $6 \mu \mathrm{g} / \mathrm{ml}$ ) or antibody (rat anti-OVA IgG, $10 \mu \mathrm{g} / \mathrm{ml}$ ) alone, each in a volume of $20 \mu \mathrm{l}$, did not induce any significant change in the mechanical (A) or thermal (B) paw withdrawal threshold $(\mathrm{n}=8)$. C-D. Changes in the PWMT (C) or PWTL (D) after injection of different doses of IgG-IC (0.1, 1 and 10 $\mu \mathrm{g} / \mathrm{ml}, 20 \mu \mathrm{l})$ or PBS $(\mathrm{n}=9)$. Data labels $(0.1$ IC, 1 IC and 10 IC) indicated the concentrations $(\mu \mathrm{g} / \mathrm{ml})$ of IgG-IC. E-F. Changes in the PWMT (E) and PWTL (F) after intra-plantar injection of IgG-IC alone $(10 \mu \mathrm{g} / \mathrm{ml}, 20 \mu \mathrm{l})$ or co-injected with the nonspecific IgG $(2 \mu \mathrm{g}$ in $20 \mu \mathrm{l})$ or Fc $\gamma \mathrm{RI}$ antibody ( $1 \mu \mathrm{g}$ in $20 \mu \mathrm{l})$, or with pre-administration of the mast cell stabilizer sodium cromoglycate (200 $\mu \mathrm{g} 20 \mu \mathrm{l}$, intra-plantar injection) or H1R blocker terfenadine (5 mg/kg, i.p.) $(\mathrm{n}=8) .{ }^{*} p<0.05$, $* * p<0.01$ vs. pre-injection mean (A-D) or IgG-IC treatment (E-F), one-way ANOVA.

Fig. 5. Hindpaw inflammation and hyperalgesia in the rat AIA model. A. The development of inflammation in mBSA-induced ankle arthritis was evaluated by the width of ankle. B. The force for lifting the hindpaw. C. Changes in the paw withdrawal mechanical threshold (PWMT). D. Changes in the paw withdrawal thermal latency (PWTL). $\mathrm{n}=6$ for each group. ${ }^{*} p<0.05, * * p<0.01$ vs. vehicle, one-way ANOVA. 
Fig. 6. Upregulation of Fc $\gamma$ RI in the DRG in the AIA model. A. qRT-PCR revealed a progressive increase in the levels of Fc $\gamma$ RI mRNA in the DRGs of AIA rats. B. Western blot revealed a significant increase in the levels of Fc $\gamma R$ I protein expression in the DRGs of AIA rats. Inset: the representative gel bands. C. Percentage of Fc $\gamma$ RI-immunopositive neuronal somata in different size categories in the DRG. D. Typical microscopic images of immunofluorescence staining for Fc $\gamma$ RI in the naïve and AIA rat. Arrows indicate Fc $\gamma R$ I-immunopositive neurons. Scale bar: $50 \mu \mathrm{m}$. N: naïve rats; S: sensitized rats (immunized but not challenged); C1-C14: AIA rats (immunized and challenged); $\mathrm{C} 1, \mathrm{C} 2, \mathrm{C} 4, \mathrm{C} 7$ and $\mathrm{C} 14$ represent the AIA rats 1 day, 2 days, 4 days, 7 days and 14 days after challenging, respectively. ${ }^{*} p<0.05, * * p<0.01$ vs. naïve, one-way ANOVA (A and B, $\mathrm{n}$ $=3$ for each group); Student's t-test ( $\mathrm{C}, \mathrm{n}=3$ for each group).

Fig. 7. Enhanced responses to IgG-IC in nociceptive DRG neurons in AIA rats. Data in A-C was from in vitro calcium imaging from dissociated DRG neurons, and data in D-F was from in vivo extracellular recordings. A. The percentage of small-sized DRG neurons responded to IgG-IC was significantly increased in AIA rats as compared with naïve rats. B. The mean magnitude of calcium responses evoked by IgG-IC was not significantly different between the naïve and AIA rats. C. The percentage of capsaicin-sensitive DRG neurons responding to IgG-IC was significantly higher in the AIA group than the naïve group. D. The percentage of neurons being evoked action potential (AP) discharges by IgG-IC injected to the peripheral receptive fields was also significantly higher in the AIA rats than in the naïve ones. E. The mechanical threshold of C-nociceptive neurons before (control) and after IgG-IC injection in the AIA and naïve rats. F. The number of action potentials evoked by mechanical stimuli of $30 \mathrm{mN}$ and $50 \mathrm{mN}$ forces (von-Frey filaments) before and after IgG-IC application in the AIA and naïve rats. Numbers in the parentheses indicated the number of neurons tested. $* p<0.05, * * p<0.01$ vs. naïve, Chi-square test (A, C and D), vs. control, paired ttest $(\mathrm{E}$ and $\mathrm{F}) ; \# p<0.05, \# \# p<0.01$ vs. naïve, t-test $(\mathrm{E}$ and $\mathrm{F})$. 

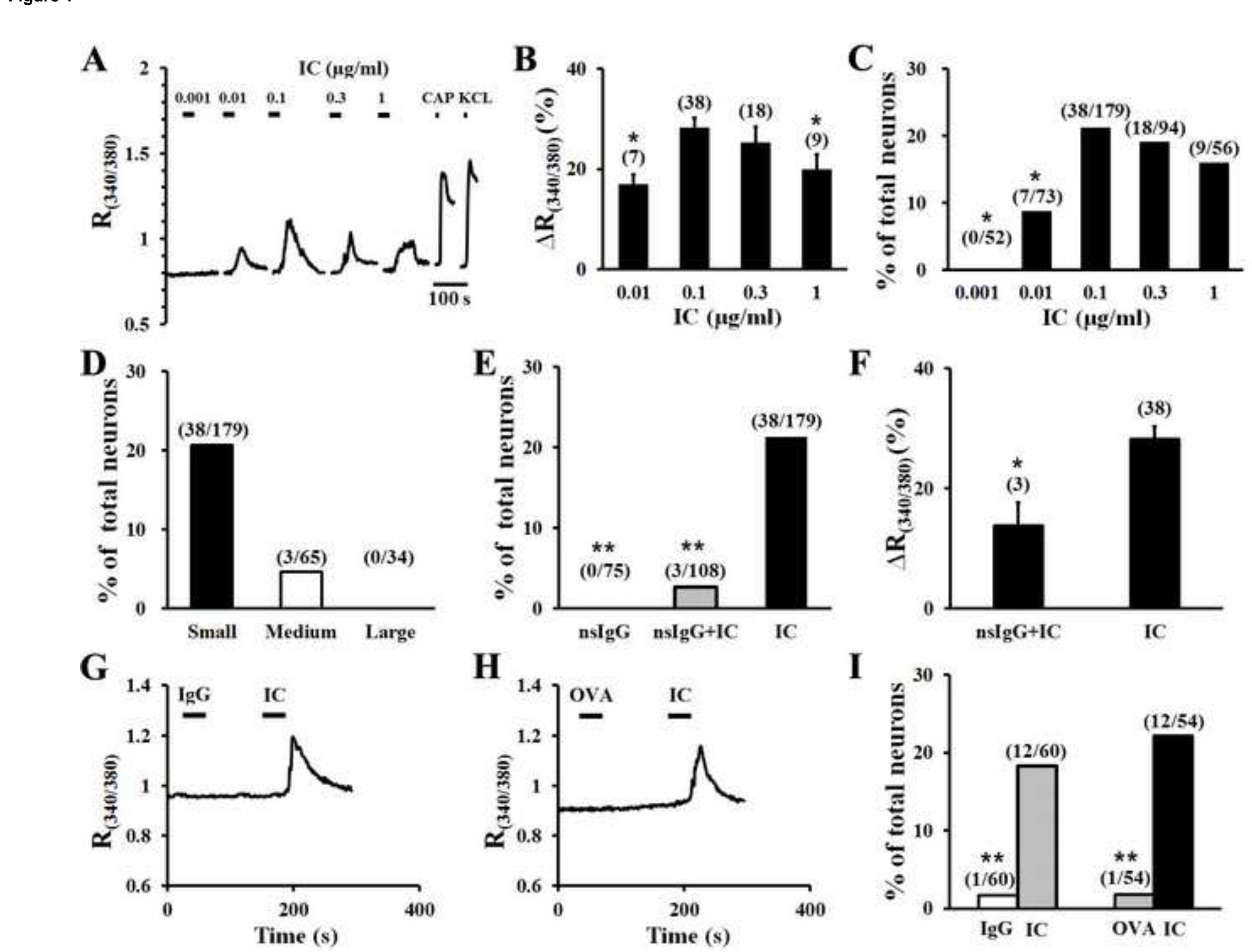
A

$10 \mathrm{mVl} \frac{\mathrm{l}}{10 \mathrm{~s}}$
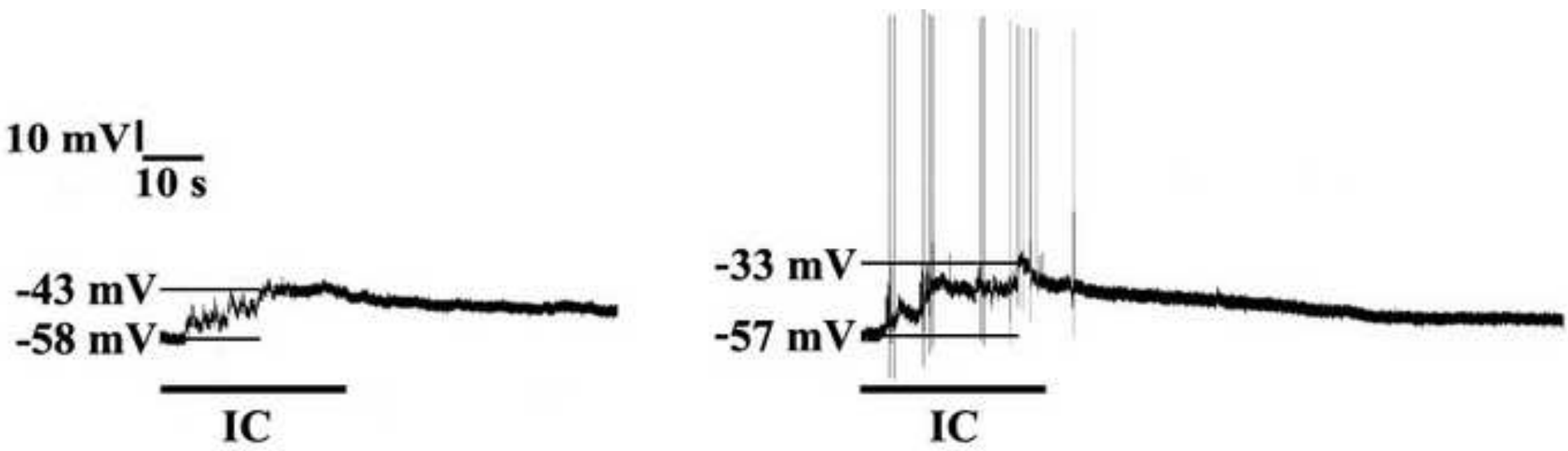

B
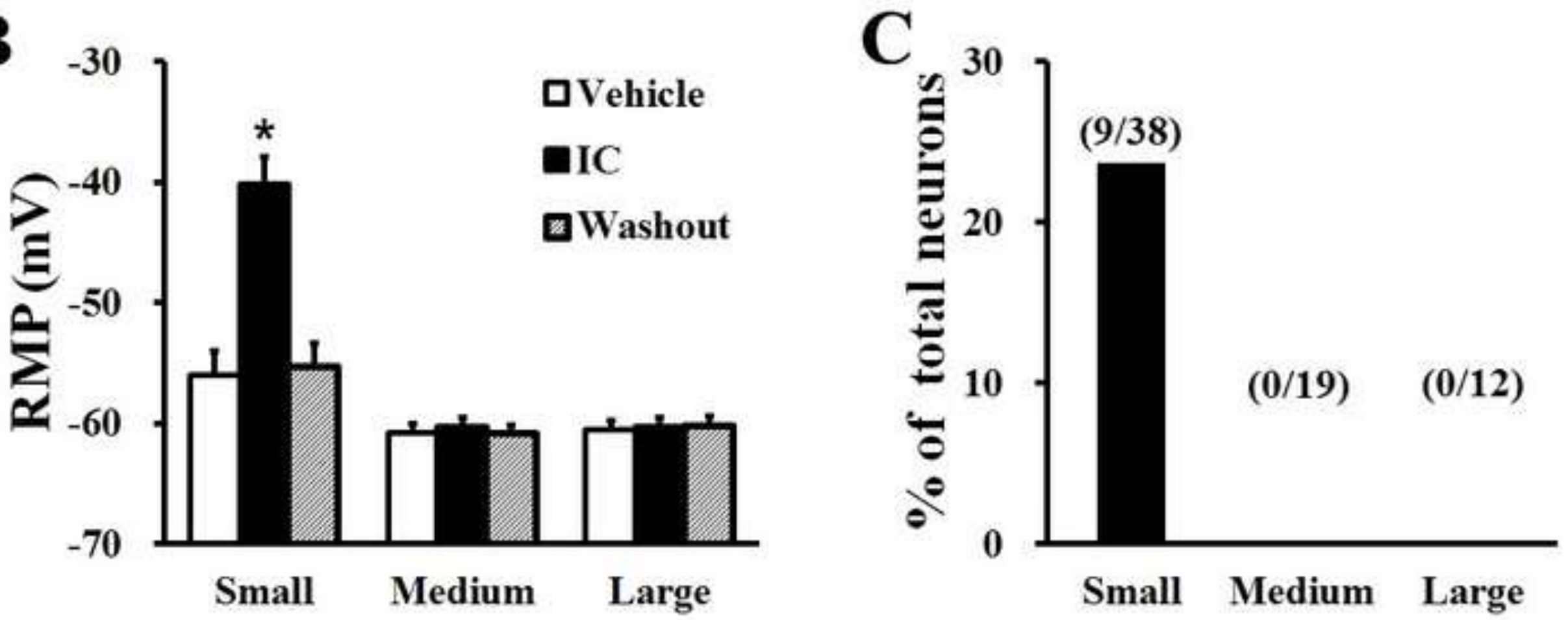

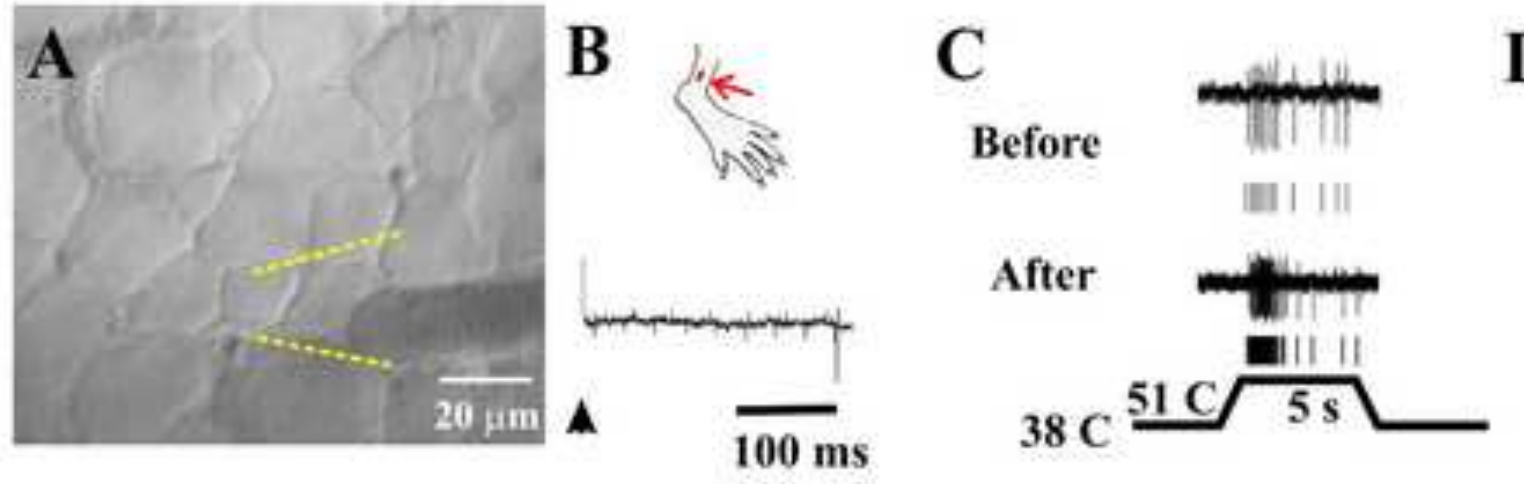

D

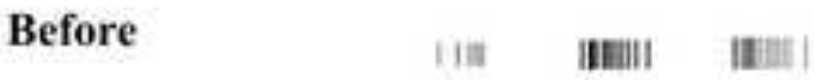

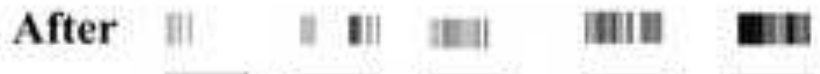

$$
\begin{aligned}
& \overline{10} \quad \overline{30} \quad \overline{50} \quad \overline{70} \overline{120 \mathrm{mN}}
\end{aligned}
$$

E i Inject

VEH

IC

$\begin{array}{lllllllllll}1 & 1 & \mid 1 & \mid 1 & \mid & 1 & \mid & 1 & 1\end{array}$

I

Time

(Min) 0

3

6 
A

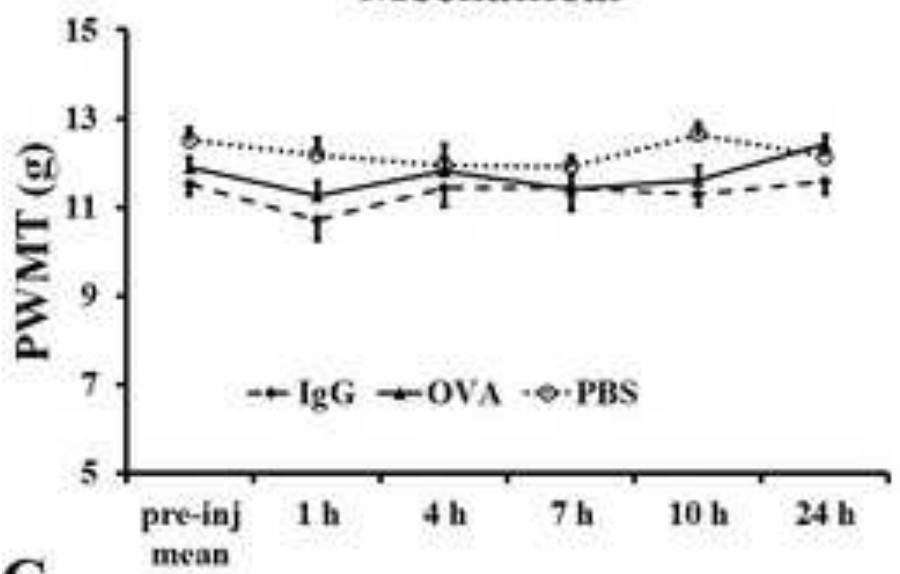

C

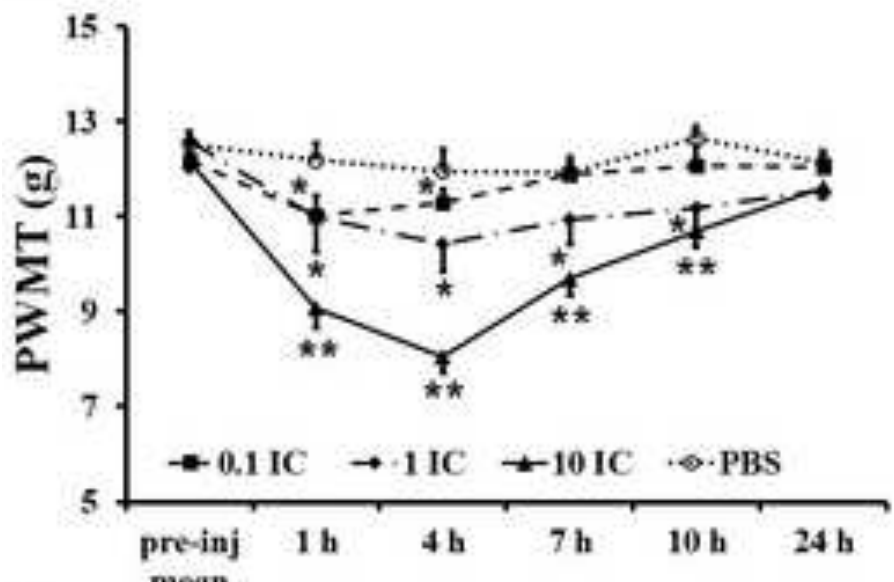

E

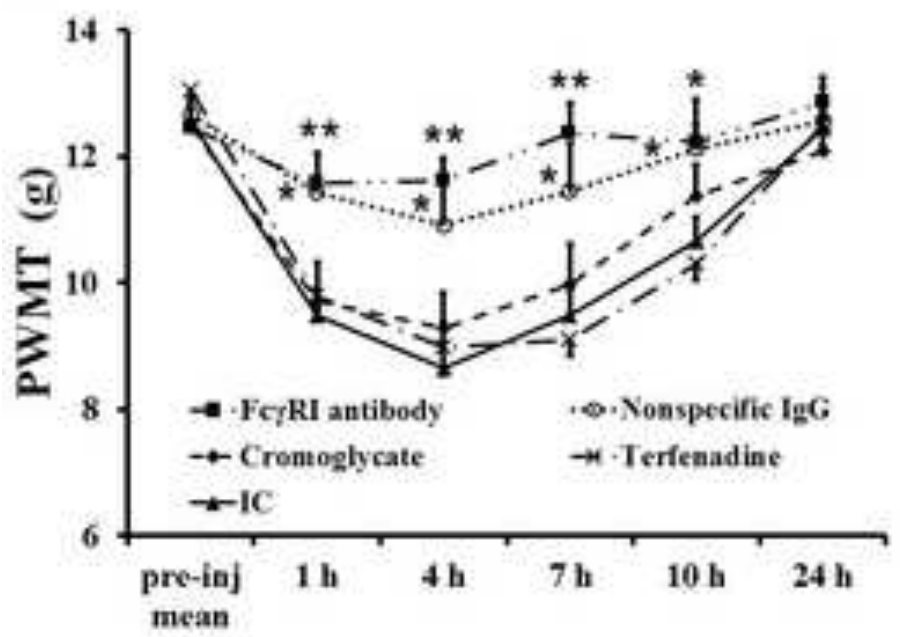

B
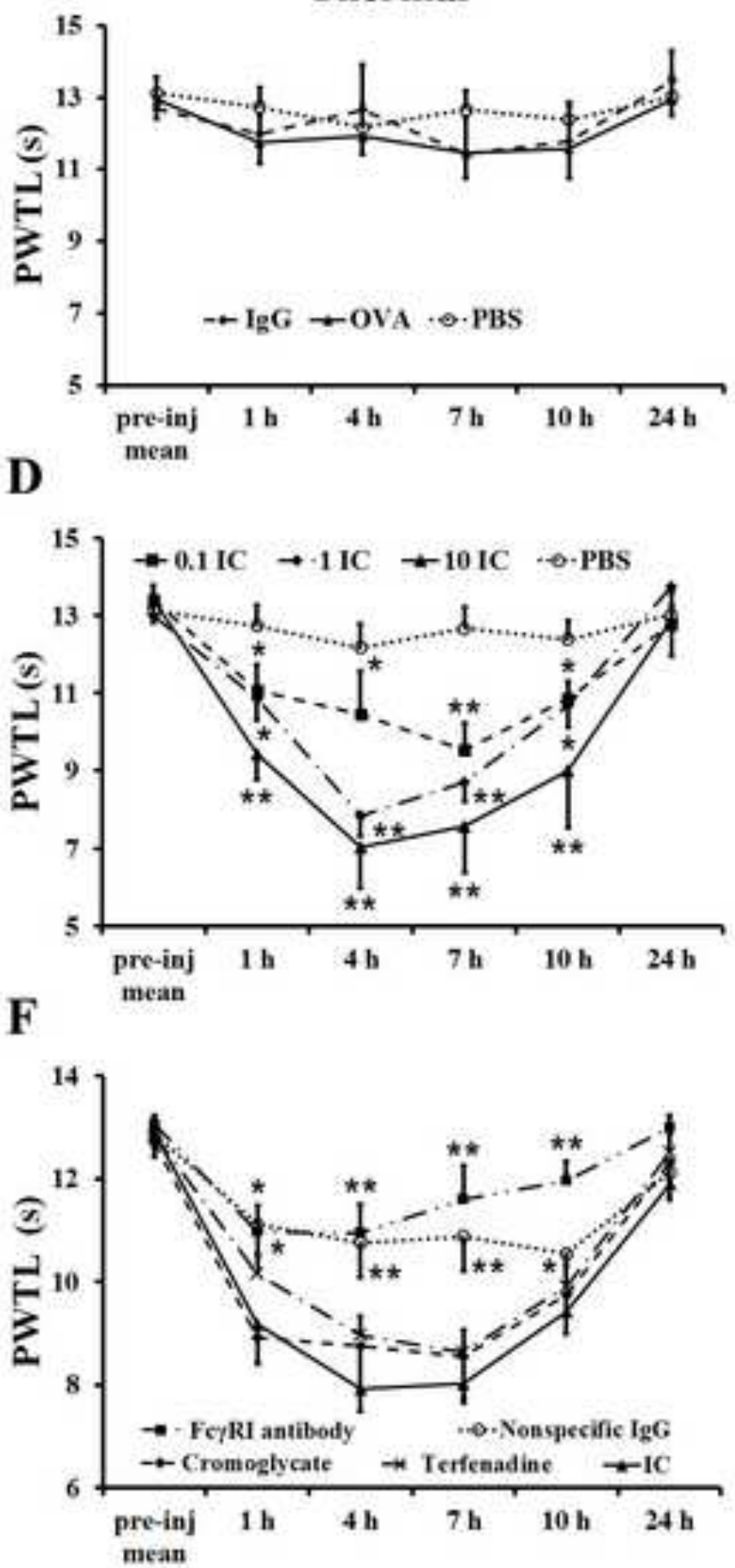

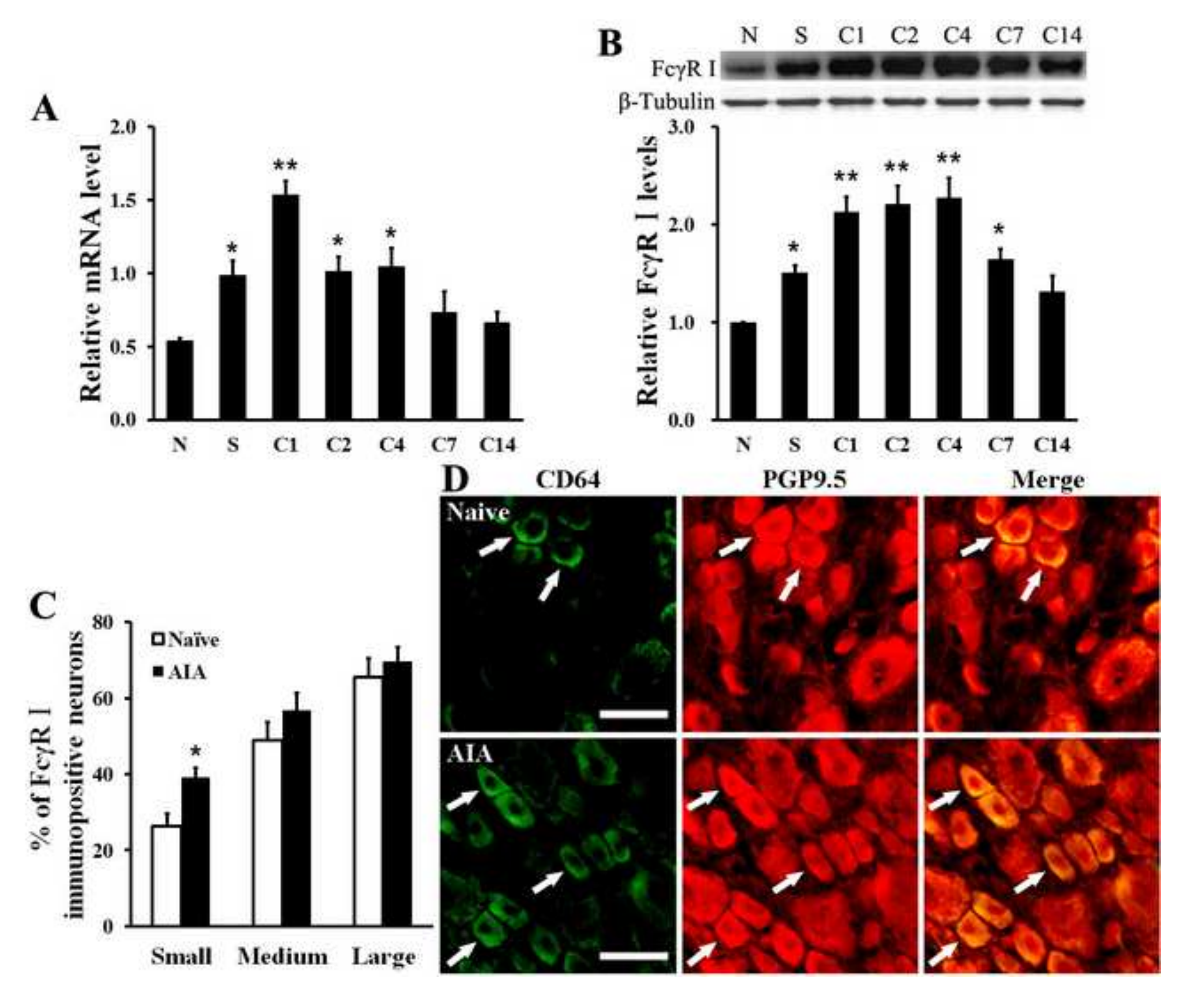

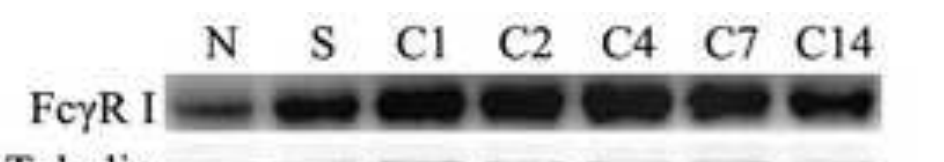

$\beta$-Tubulin $--\infty-$

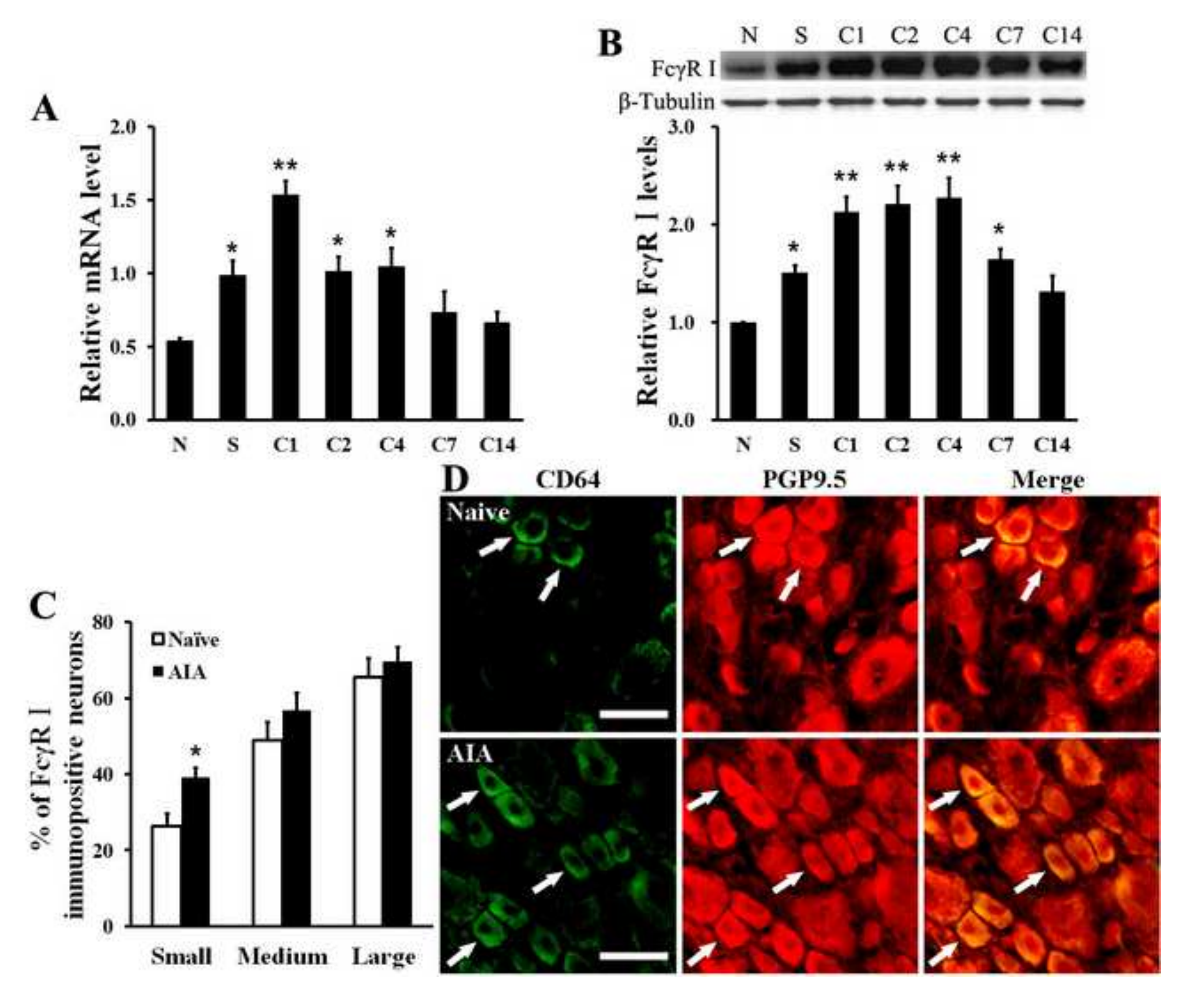

\section{B \\ B}

A

\section{Fing}

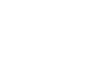


